\title{
The Resistive Switching Behavior of Al/Chitosan-Graphene Oxide/FTO Structure
}

\author{
Hau Huu Do Ho, ${ }^{1,2}$ Trung Minh Le, ${ }^{1,2}$ and Ngoc Kim Pham $\mathbb{D}^{1,2}$ \\ ${ }^{1}$ Faculty of Materials Science and Technology, University of Science, Ho Chi Minh City, Vietnam \\ ${ }^{2}$ Vietnam National University, Ho Chi Minh City, Vietnam \\ Correspondence should be addressed to Ngoc Kim Pham; phamkngoc@hcmus.edu.vn
}

Received 19 February 2021; Revised 12 March 2021; Accepted 18 March 2021; Published 7 April 2021

Academic Editor: Nguyen Duc Cuong

Copyright (C) 2021 Hau Huu Do Ho et al. This is an open access article distributed under the Creative Commons Attribution License, which permits unrestricted use, distribution, and reproduction in any medium, provided the original work is properly cited.

\begin{abstract}
Resistive random access memory (RRAM) is emerging as a new class of nonvolatile memory that offers promising electronic properties and simple metal-insulator-metal (MIM) structures for sandwich layers, such as organics, inorganics, and hybrid materials. Hybrid structures have attracted much interest recently because of their advantageous properties. The combination of chitosan (CS) and graphene oxide (GO) acts as switching layers in the Al/CS-GO/FTO RRAM structure it is studied with bipolar switching behavior at approximately $10^{2}$ ON/OFF ratios during 100 cycles. This hybrid interaction is identified by shifts in the $\mathrm{D}, \mathrm{G}$, and $2 \mathrm{D}$ bands using Raman spectroscopy. The conduction mechanism is proposed to be a space-charge-limited conduction (SCLC) mechanism and trap-assisted tunneling conduction mechanism in the ON and OFF states, respectively. The trapped and detrapped electrons move through the trap sites with external electric fields, and this movement is responsible for the switching mechanism of the CS-GO nanocomposite memory device.
\end{abstract}

\section{Introduction}

There has been a recent rapid increase in the field of nanotechnology, particularly in memory storage devices [1]. Increased demands for information-storage have required the development of new technologies to meet these demands. Resistive random access memory (RRAM) is one candidate for next-generation information storage due to its high speed, long retention time with low power consumption, and simple structure $[2,3]$. Different materials such as organics, inorganics, transition metal dichalcogenides, metal-organic frameworks, and hybrid materials [4-12] have been utilized in RRAM devices to demonstrate their potential applications in data storage.

Environmentally friendly materials such as chitin/chitosan, cellulose, alginate, and carbohydrate polymers [13] have recently received much attention due to their biodegradability, antibacterial properties, protein affinity, and biocompatibility $[1,14]$. Chitosan is a component of the protective shell of marine crustaceans, such as crabs, shrimps, and crayfish. Chitosan is composed of bonded $\beta(1 \longrightarrow 4)-2$ amino-2-deoxy-D-glucopyranoses and $\beta(1 \longrightarrow 4)$-2-aceta-
mido-2-deoxy-D-glucopyranoses [1]. It is chemically flexible because there are three types of functional groups in the polymers, which are amines, acetamides, and both primary and secondary hydroxyl groups. With these advantages, the use of chitosan is increasing [1]. Currently, chitosan is sold as a white powder that can be dissolved in dilute organic acids, such as acetic, lactic, hydrochloric, formic, or succinic acid [15]. However, it is a poor conductor so it is necessary to add a conductive filler in data storage applications [16].

Two-dimensional graphene oxide (GO) nanosheets have recently been synthesized from graphite. It has an advantageous chemical structure that leads to improved mechanical properties, biological compatibility, water superiority, low cost, lightweight, and excellent durability [17, 18]. Therefore, GO has been focused on changing the thickness and control the number of functional groups with organic compounds. This opens it as a promising biomaterial that is available in nature.

We studied resistive memory devices in capacitor-like $\mathrm{Ag} / \mathrm{CS}-\mathrm{GO} / \mathrm{FTO}$ structures in a previous report where the CS-GO nanocomposites acted as the memory layer [19]. The devices showed a bipolar resistive-switching effect under 
an external electric field with an endurance of $10^{3}$ and an ON/OFF ratio of $10^{2}$. We have changed the top electrode from $\mathrm{Ag}$ to $\mathrm{Al}$ to demonstrate the stability of the resistive switching behavior of the CS-GO nanocomposites. The $\mathrm{Al} / \mathrm{CS}-\mathrm{GO} / \mathrm{FTO}$ structure was fabricated using a CS-GO nanoblend to act as a switching layer to improve the stability and conductivity. The GO was used to enhance the resistive switching behavior and CS to make it more environmentally friendly. The electrical conductivity and resistive switching mechanisms of Al/CS-GO/FTO were also analyzed. This study is aimed at improving our understanding of resistive switching in CS-GO for RRAM applications.

\section{Experimental Procedure}

2.1. Materials. The $4-10 \%$ edge-oxidized GO powders were supplied by Sigma Aldrich (average of 15-20 sheets). The CS powders ( $\mathrm{MW}=100,000-300,000$, degree of deacetylation of $76 \%$ ) and acetic acid (99.5\% purity) were purchased from Acros and used to synthesize the CS-GO nanocomposite. The commercial FTO-coated glass (MTI, USA) with a resistance of $12 \Omega$ was used for device fabrication.

2.2. Fabrication of Devices. A $0.5 \mathrm{mg} / \mathrm{mL}(\sim 0.05 \% \mathrm{wt} / v) \mathrm{GO}$ solution was prepared in deionized water and stirred for six hours. The CS powder was dissolved in acetic acid to create a $2 \% \mathrm{wt} / v$ solution and stirred for 12 hours. These two solutions were mixed at a 1:1 ratio and stirred for one hour at $60^{\circ} \mathrm{C}$ to create a homogeneous solution.

The CS-GO thin film was coated on an FTO substrate by spin coating at $3000 \mathrm{rpm}$ for 60 seconds and then heattreated at $50^{\circ} \mathrm{C}$ to evaporate the solvent. The membrane was annealing for 12 hours to improve the stability. Finally, the Al electrode was deposited by evaporation using a shadow mask with a target-substrate spacing of $10 \mathrm{~cm}$ (Figure 1).

2.3. Analysis Technique. A semiconductor characterization system (Keithley 2400) and probe station were used to perform current-voltage measurements. A bias sweep sequence of $0 \mathrm{~V} \longrightarrow-7 \mathrm{~V} \longrightarrow 0 \mathrm{~V} \longrightarrow 7 \mathrm{~V} \longrightarrow 0 \mathrm{~V}$ was applied to the memory device. Raman spectra (Labram 300, Horiba) were used to analyze scattering oscillations in the CS, GO, and CS-GO nanocomposites. The morphology of GO was studied by using the transmission electron microscopy technique with a JEOL JEM-2100F system. The X-ray photoelectron spectroscopy (XPS) was conducted for the chemical state analysis by the Thermo Fisher Scientific (UK) system with a monochromated source of $\mathrm{Al} \mathrm{K}_{\alpha}(h v=1486.6 \mathrm{eV})$.

\section{Results and Discussion}

3.1. Structural Characteristics. Figure 2 shows the Raman spectra of CS, CS-GO, and GO. Three peaks were observed in the GO spectrum and labeled as D band at $\sim 1329 \mathrm{~cm}^{-1}$, $\mathrm{G}$ band at $\sim 1570 \mathrm{~cm}^{-1}$, and $2 \mathrm{D}$ band at $\sim 2656 \mathrm{~cm}^{-1}[8,9]$. The $\mathrm{D}$ band is attributed to defects and disturbances in the hexagonal graphite layers, which is characteristic of the inplane stretching motion of $\mathrm{sp}^{2}$ carbon atoms [10]. The Gband appeared due to defects, deformations, doping, and temperature. It was used to detect the reactions of graphene-based materials [8]. The 2D band was used to characterize changes in the number of GO layers. The 2D peak of CS-GO shifted from $2656 \mathrm{~cm}^{-1}$ to $2682 \mathrm{~cm}^{-1}$ in the GO and CS-GO spectra, which showed the change in the GO layer as it was embedded into the CS matrix [11]. The features of both CS and GO were detected in the CS-GO spectrum. The bands D, G, and 2D of CS-GO are all higher compared to GO. Shifts from $2656 \mathrm{~cm}^{-1}$ to $2682 \mathrm{~cm}^{-1}$ for band $2 \mathrm{D}$, $1329 \mathrm{~cm}^{-1}$ to $1336 \mathrm{~cm}^{-1}$ for band $\mathrm{D}$, and $1570 \mathrm{~cm}^{-1}$ to $1586 \mathrm{~cm}^{-1}$ for band $\mathrm{G}$ indicate the physical and chemical interactions between the GO and CS functional groups [12].

3.2. Surface Morphology. Figure 3 shows TEM images of the GO morphology at a scale of $200 \mathrm{~nm}$ and $100 \mathrm{~nm}$. It is observed that the GO has various size clusters and fairly evenly dispersed. Uncontrolled temperature or oxidation could be responsible for the irregular shapes and uneven particle size [20]. GO was distributed into a thin flat sheet on a surface and folds to create wrinkles [21]. The solid graphene sheets are affected by structural disturbances, possibly due to the laminated thin layers and crumpled sheets involved during exfoliation in a reducing and saponification medium with the removal of oxygen alternating other functional groups between layers [20].

\subsection{Elemental Composition and Oxidation States of ZnO NPs.} $\mathrm{X}$-ray electron spectroscopy (XPS) was used to determine the chemical states of various elements and the presence of functional groups. The scan spectrum showed that the main elements are carbon and oxygen (Figure 4(a)). The peaks identified in the GO spectrum at $\sim 284.4 \mathrm{eV}$ and $\sim 531.8 \mathrm{eV}$ are the $\mathrm{C} 1 \mathrm{~s}$ and $\mathrm{O}$ 1s excitations, respectively. Figure 4(b) shows the convoluted $\mathrm{C} 1 \mathrm{~s}$ peak, including different binding energies of 284.5, 284.8, 286.5, 287.5, and $288.9 \mathrm{eV}$. These peaks correspond to different functional groups of $\mathrm{sp}^{2} \mathrm{C}=\mathrm{C}$ (42.9\%) and $\mathrm{sp}^{3} \mathrm{C}-\mathrm{C}(47.6 \%)$ in the aromatic rings, along with $\mathrm{C}-\mathrm{O}(4.7 \%), \mathrm{C}=\mathrm{O}(1.2 \%)$, and $\mathrm{O}=\mathrm{C}-\mathrm{OH}(3.6 \%)$ [22]. The convoluted $\mathrm{O}$ 1s peak (Figure 4(c)) shows two peaks at binding energies of 533.1 and $533.4 \mathrm{eV}$, which are assigned to $\mathrm{C}-\mathrm{O}$ bonds $(66.4 \%)$ and $\mathrm{C}=\mathrm{O}$ bonds $(33.6 \%)$, respectively [23]. These two peaks correspond to the oxygen in the surface hydroxyl groups of GO. The atomic ratio of carbon to oxygen $(\mathrm{C} / \mathrm{O})$ was determined from XPS to be 20 , which indicates that there are oxygen vacancies in the surface of GO layers. These vacancies can act as traps that support the electrical conduction and resistive switching mechanism of the memory device.

3.4. Resistive Switching Characteristics. A bias sweep in a sequence of $0 \mathrm{~V} \longrightarrow-7 \mathrm{~V} \longrightarrow 0 \mathrm{~V} \longrightarrow 7 \mathrm{~V} \longrightarrow 0 \mathrm{~V}$ was applied to the devices. The $I-V$ characteristics are shown in Figure 5 with a current threshold of $0.5 \mathrm{~mA}$ applied to prevent the breakdown of the device [24]. The device was initially in a high-resistance state (HRS) with no bias applied. The high conductivity ON state was achieved by applying negative voltage. The current increased gradually with increasing negative voltage and jumped quickly at approximately $-3 \mathrm{~V}$, converting the device to a low-resistance state (LRS). This transition from HRS to LRS is referred to as the 


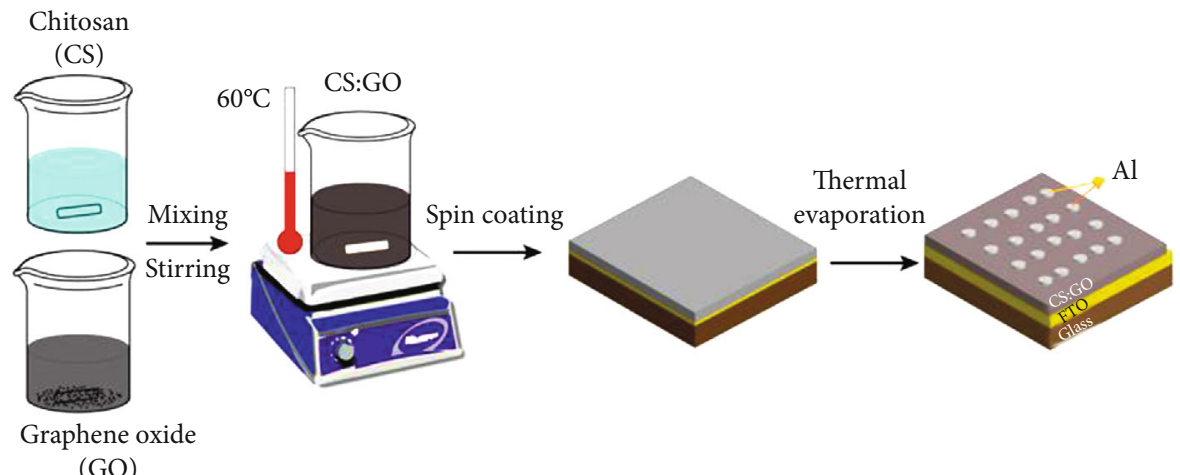

(GO)

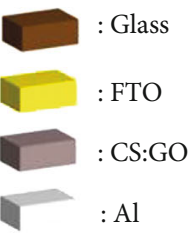

FIgURE 1: Schematic diagram of the fabrication process of the Al/CS-GO/FTO device.

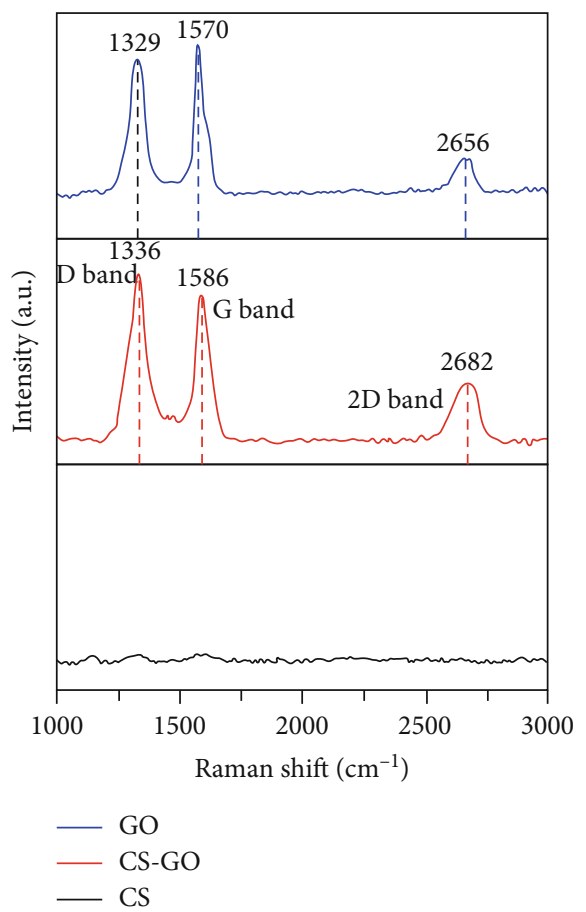

FIGURE 2: Raman spectra of the CS, CS-GO, and GO.

"writing" process or the SET process, and this state was retained during the voltage sweep back from $-7 \mathrm{~V}$ to $0 \mathrm{~V}$. The current dropped suddenly around $+1 \mathrm{~V}$ when applying a positive voltage, which indicates the resistive switching from LRS to HRS and referred to as the "erase" process or RESET process. These results show that the clockwise bipolar resistive switching behavior with the SET state was due to the negative voltage, and the positive voltage was specified as the RESET state.
Figures 6(a) and 6(b) show the endurance and cumulative probability performance of the Al/CS-GO/FTO structure at $V_{\text {read }}=-0.5 \mathrm{~V}$. The switching behavior was maintained with an "ON/OFF" ratio of approximately $10^{2}$ during 100 cycles. However, both stages fluctuate little. The variation of the operating voltages is shown in Figure 6(c). The SET voltages ranged from $-3 \mathrm{~V}$ to $-4 \mathrm{~V}$ while the RESET voltages ranged from $0.7 \mathrm{~V}$ to $1.5 \mathrm{~V}$ between devices. The RESET voltage deviates less compared to the SET voltage in each device. The SET and RESET voltages are in the opposite polarities so we classify this memory device as a bipolar resistive switch. This bipolar behavior avoids confusion during the operation.

The experimental $I-V$ curve was converted to the $J-E$ plot shown in Figure 7 to understand the conduction mechanism in the process of the Al/CS-GO/FTO device. The plot of $J$ versus $E$ from $0 \mathrm{~V}$ to $-7 \mathrm{~V}$ is consistent with a space-chargelimited conduction (SCLC) mechanism as shown in Figure 7 (a) with three distinct curves of either ohmic $(I \sim V)$, space-charge-trap-filled limit $\left(V_{\mathrm{TFL}}\right)\left(I \sim V^{n}\right.$ with $\left.n>2\right)$, or Child's $\left(I \sim V^{2}\right)$, which are given by equations (1)-(3) [25-27]:

$$
\begin{gathered}
J_{\text {ohmic }}=q n_{o} \mu \frac{V}{d}, \\
J_{\mathrm{TFL}}=\frac{9}{8} \mu \varepsilon \theta \frac{V^{2}}{d^{3}}, \\
J_{\text {Child }}=\frac{9}{8} \mu \varepsilon \frac{V^{2}}{d^{3}} .
\end{gathered}
$$

Ohmic conduction was observed in the voltage range from $0 \mathrm{~V}$ to $-3 \mathrm{~V}$, corresponding to a slope of approximately one $\left(I \sim V^{1.27}\right)$. This can be attributed to thermally generated carriers that are more dominant than injected carriers in the dielectric layer [27]. Alternatively, the current was the dominant control in the trap-filled limit with a slope of $I \sim V^{120}$ 


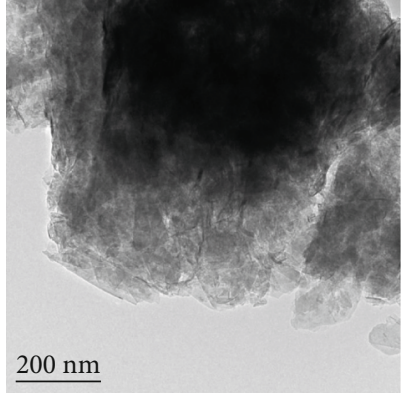

(a)

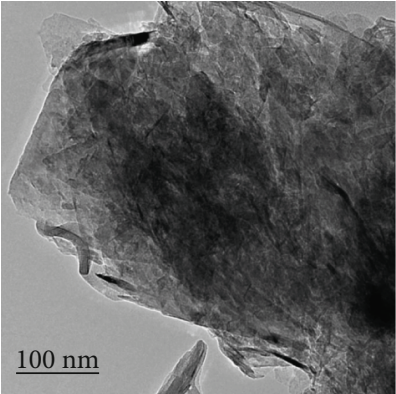

(b)

Figure 3: TEM images of the GO sheets at a scale of (a) $200 \mathrm{~nm}$ and (b) $100 \mathrm{~nm}$.

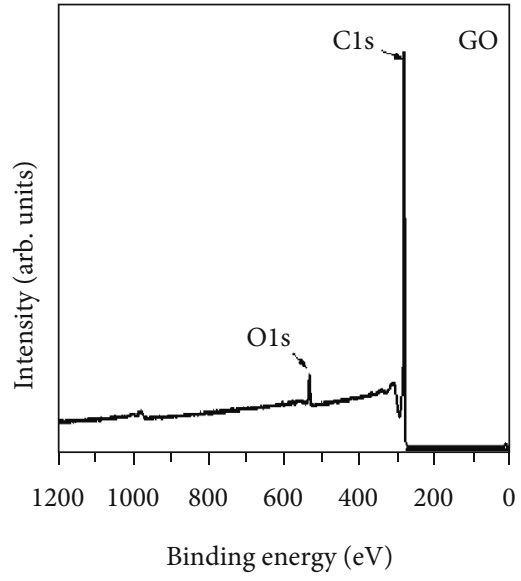

(a)

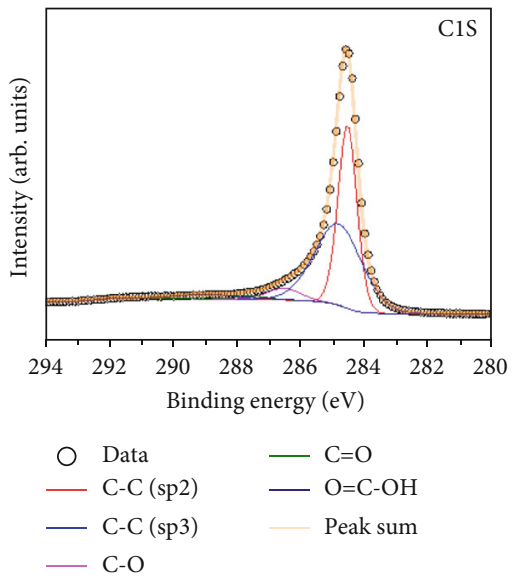

(b)

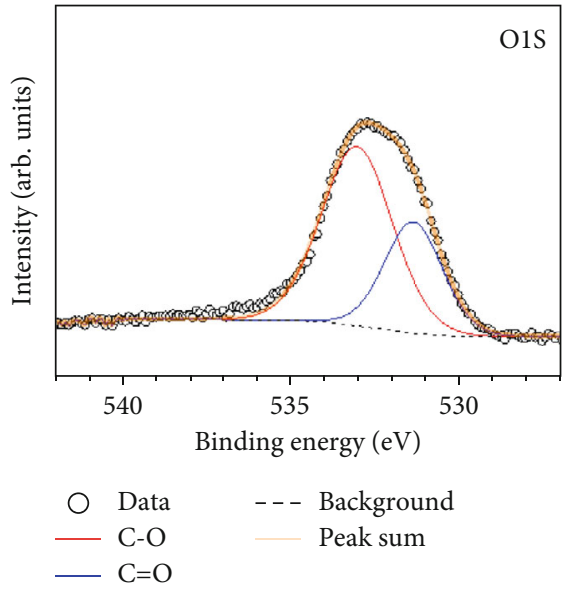

(c)

Figure 4: The X-ray photoelectron survey spectrum of GO (a), the convoluted C 1s high-resolution spectrum (b), and the convoluted O 1s spectrum of GO (c).

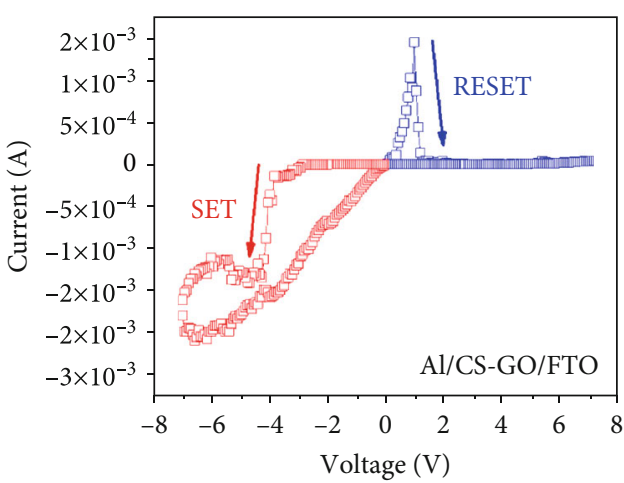

(a)

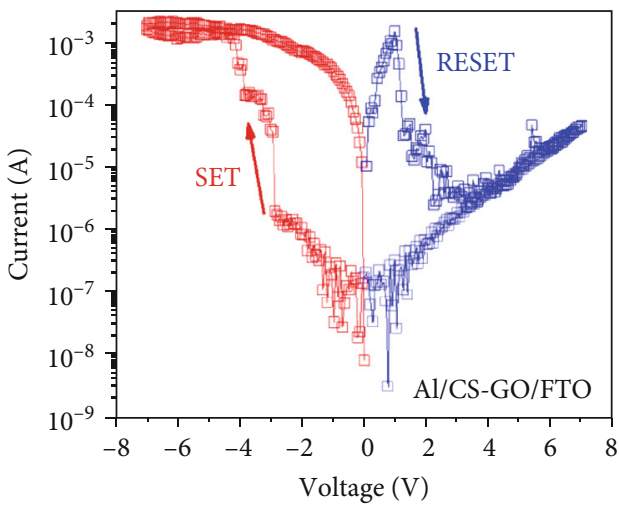

(b)

FIGURE 5: A linear (a) and semilog (b) plot of the typical current-voltage ( $I-V)$ characteristics of an Al/CS-GO/FTO device.

because of the free movement of the carriers in the dielectric layer after all traps are filled [26]. The slope decreased gradually to $I \sim V^{2}$ due to the accumulation of a space-charge layer in the dielectric layer from $-3.5 \mathrm{~V}$ to $-4 \mathrm{~V}$ [28].
The current was consistent with variable-range hopping (VRH) in the back sweep from $-7 \mathrm{~V}$ to $0 \mathrm{~V}$, which is shown in Figure 7(b). The relationship between $J$ versus $E$ is given by 


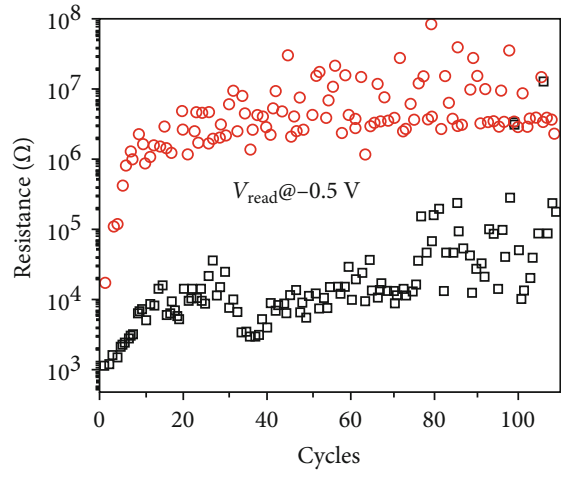

$\square$ LRS

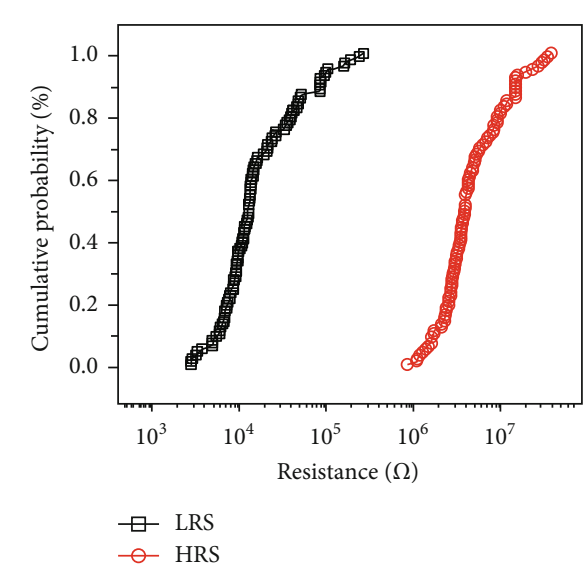

$\square$ LRS

(b)

(a)

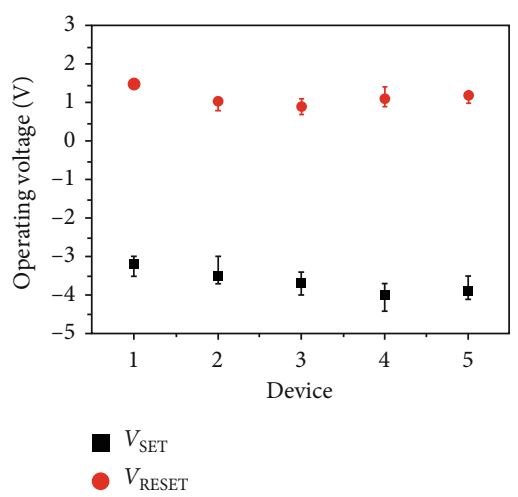

(c)

FIGURE 6: The endurance performance (a) and cumulative probability test (b) at 100 cycles under $V_{\text {read }}=-0.5 \mathrm{~V}$. The variation of operating voltages in five devices of $\mathrm{Al} / \mathrm{CS}-\mathrm{GO} / \mathrm{FTO}(\mathrm{c})$.

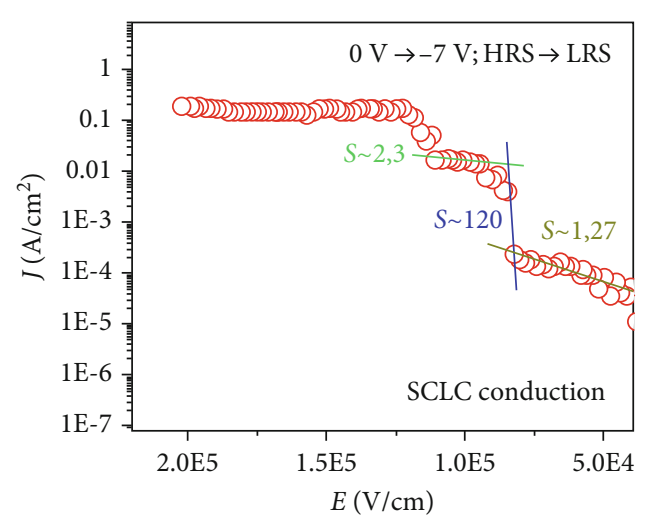

(a)

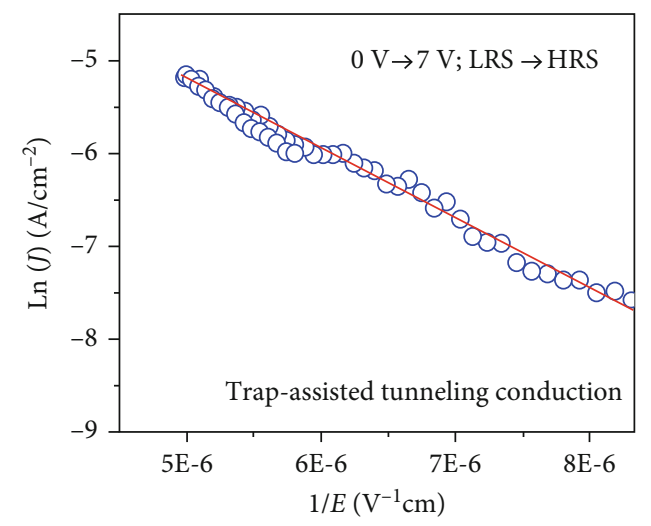

(c)

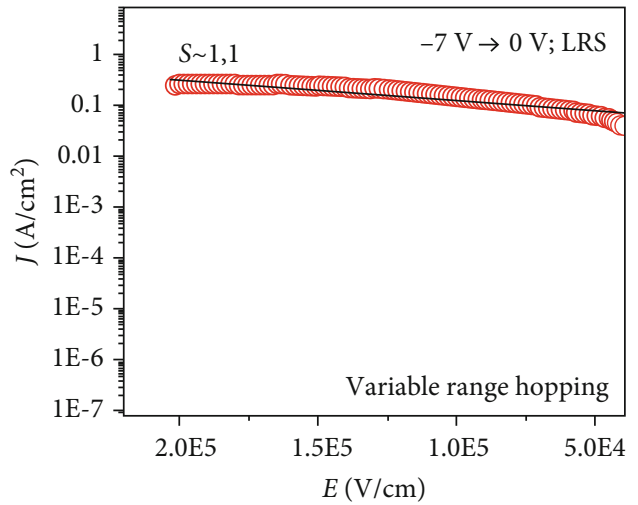

(b)

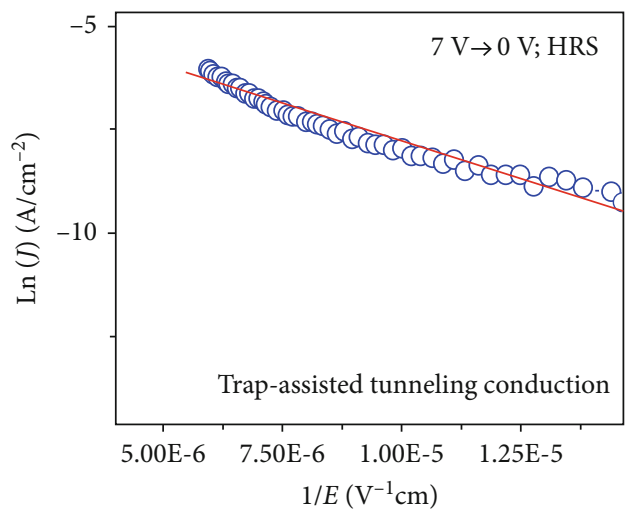

(d)

Figure 7: The electrical conduction mechanism of the Al/CS-GO/FTO structure in SCLC conduction (a), variable range hopping (b), and trap-assisted tunneling conduction (c, d).

$$
J_{\mathrm{VRH}}=\sigma_{0} \exp \left(\frac{-T_{0}}{T}\right)^{1 / 4} E .
$$

Here, $\sigma_{0}$ is the electrical conductivity at $T_{0}$. The temperatures $T_{0}$ and $T$ are the absolute temperatures, and all other variables are the same as defined previously. The slope $J \sim E$ about 1.1 is likely influenced by the hopping of electrons in the thin-film layer through traps localized in the charge-carrier states [25].

The current dropped abruptly at $V_{\text {reset }}$ of $1 \mathrm{~V}$ during the reverse voltage from $0 \mathrm{~V}$ to $7 \mathrm{~V}$. The current at HRS is 
predominantly controlled by trap-assisted tunneling conduction as represented by

$$
J_{\mathrm{TAT}}=A \exp \left(\frac{-8 \pi \sqrt{2 q m^{*}}}{3 h E} \varphi_{T}^{3 / 2}\right)
$$

Here, $A$ is a constant, $m^{*}$ is effective mass of an electron, $h$ is Planck's constant, and $\varphi_{T}$ is the energy of the electron traps. All other variables are the same as defined before.

Based on these results, we proposed that the resistive switching mechanism in Al/CS-GO/FTO is due to the charge trapping and detrapping process at the defect sites in composite CS-GO. GO usually has many structural defects due to the manufacturing process [29] including oxygen vacancies as shown in the XPS spectra, which act as trapping positions in the CS-GO matrix. Injected electrons from the $\mathrm{Al}$ electrode to CS-GO may undergo two processes simultaneously when a negative voltage is initially applied. First, some electrons move freely by hopping between trapped positions into the matrix heading to the FTO electrode. Second, the other electrons are held in trapped positions. There is subsequently an increase in the number of electrons that move directly to the bottom electrode without any resistance forming a continuous current when all traps are filled. This switched the device to the ON state with a high conductivity current. Electrons injected from the FTO electrode still move freely in a dielectric layer when the positive voltage is applied until the voltage is high enough to disrupt the conducting channels which switched the device to the ON state.

\section{Conclusion}

In summary, we have successfully combined CS and GO in an $\mathrm{Al} / \mathrm{CS}-\mathrm{GO} / \mathrm{FTO}$ structure with bipolar along with repeatable and reliable reversible resistive switching abilities. The CS-GO acts as a switching layer with an ON/OFF ratio of $\sim 10^{2}$ over 100 cycles. The conduction mechanism which was related to SCLC, Ohm's law, VRH, and trap-assisted tunneling conduction mechanisms were demonstrated to exist in the bias sweeps. The resistive switching was activated by trapping/detrapping electrons in the CS-GO layer under the external electric field due to the existence of oxygen vacancies in GO. This CS-GO hybrid material shows promise as an environmentally friendly memory device.

\section{Data Availability}

Data is available within the article.

\section{Conflicts of Interest}

There are no conflicts of interest to declare.

\section{Acknowledgments}

This research is funded by Viet Nam National University Ho Chi Minh City (VNU-HCM) under grant number C2018-18-27.

\section{References}

[1] K. Pieklarz, M. Tylman, and Z. Modrzejewska, "Applications of chitosan-graphene oxide nanocomposites in medical science: a review," Progress on Chemistry and Application of Chitin and its Derivatives, vol. 23, pp. 5-24, 2018.

[2] T. C. Chang, K. C. Chang, T. M. Tsai, T. J. Chu, and S. M. Sze, "Resistance random access memory," Materials Today, vol. 19, no. 5, pp. 254-264, 2016.

[3] F. Zahoor, T. Z. Azni Zulkifli, and F. A. Khanday, "Resistive random access memory (RRAM): an overview of materials, switching mechanism, performance, multilevel cell (mlc) storage, modeling, and applications," Nanoscale Research Letters, vol. 15, no. 1, p. 90, 2020.

[4] H. Wang and X. Yan, "Overview of resistive random access memory (RRAM): materials, filament mechanisms, performance optimization, and prospects," physica status solidi (RRL) - Rapid Research Letters, vol. 13, no. 9, p. 1900073, 2019.

[5] Z. Shen, C. Zhao, Y. Qi et al., "Advances of RRAM devices: resistive switching mechanisms, materials and bionic synaptic application,” Nanomaterials, vol. 10, no. 8, p. 1437, 2020.

[6] L. Zhu, J. Zhou, Z. Guo, and Z. Sun, “An overview of materials issues in resistive random access memory," Journal of Materials, vol. 1, no. 4, 295 pages, 2015.

[7] S. Munjal and N. Khare, "Multilevel resistive and magnetization switching in $\mathrm{Cu} / \mathrm{CoFe} 2 \mathrm{O} 4 / \mathrm{Pt}$ device: coexistence of ionic and metallic conducting filaments," Applied Physics Letters, vol. 113, no. 24, article 243501, 2018.

[8] A. Nife and O. F. T. O. Device, "Forming free resistive switching characteristics," in AIP Conference Proceedings, p. 020171, Greece, 2020.

[9] J. Molina-Reyes and L. Hernandez-Martinez, "Understanding the resistive switching phenomena of stacked $\mathrm{Al} / \mathrm{Al} 2 \mathrm{O} 3 / \mathrm{Al}$ thin films from the dynamics of conductive filaments," Complexity, vol. 2017, 10 pages, 2017.

[10] D. Filatov, I. Kazantseva, D. Antonov et al., "Conductive atomic force microscopy study of the resistive switching in yttria- stabilized zirconia films with Au nanoparticles," Scanning, vol. 2018, 9 pages, 2018.

[11] S. Munjal and N. Khare, "Electroforming free controlled bipolar resistive switching in $\mathrm{Al} / \mathrm{CoFe} 2 \mathrm{O} 4 / \mathrm{FTO}$ device with self-compliance effect," Applied Physics Letters, vol. 112, no. 7, article 073502, 2018.

[12] S. Munjal and N. Khare, "Compliance current controlled volatile and nonvolatile memory in $\mathrm{Ag} / \mathrm{CoFe} 2 \mathrm{O} 4 / \mathrm{Pt}$ resistive switching device," Nanotechnology, vol. 32, no. 18, p. 185204, 2021.

[13] V. K. Thakur, M. K. Thakur, P. Raghavan, and M. R. Kessler, "Progress in green polymer composites from lignin for multifunctional applications: a review," ACS Sustainable Chemistry \& Engineering, vol. 2, no. 5, pp. 1072-1092, 2014.

[14] A. Ali and S. Ahmed, "A review on chitosan and its nanocomposites in drug delivery," International Journal of Biological Macromolecules, vol. 109, pp. 273-286, 2018.

[15] M. H. Periayah, A. S. Halim, and A. Z. M. Saad, "Chitosan: a promising marine polysaccharide for biomedical research," Pharmacognosy Reviews, vol. 10, no. 19, pp. 39-42, 2016.

[16] A. Barra, N. M. Ferreira, M. A. Martins et al., "Eco-friendly preparation of electrically conductive chitosan - reduced graphene oxide flexible bionanocomposites for food packaging 
and biological applications," Composites Science and Technology, vol. 173, pp. 53-60, 2019.

[17] J. Ahmed, M. Mulla, Y. A. Arfat, and T. Lidia Arockia Thai, "Mechanical, thermal, structural and barrier properties of crab shell chitosan/graphene oxide composite films," Food Hydrocolloids, vol. 71, pp. 141-148, 2017.

[18] N. Raeis-Hosseini and J. S. Lee, "Resistive switching memory using biomaterials," Journal of Electroceramics, vol. 39, no. 14, pp. 223-238, 2017.

[19] K. My Tran, D. Phuc Do, H. Nam Vu et al., "Experimental combined theoretical study on chemical interactions of graphene oxide with chitosan and its resistive-switching effect," Materials Science and Engineering: B, vol. 262, article 114788, 2020.

[20] M. Aziz, F. S. Abdul Halim, and J. Jaafar, "Preparation and characterization of graphene membrane electrode assembly," Jurnal Teknologi, vol. 69, no. 9, pp. 11-14, 2014.

[21] A. K. Mishra and S. Ramaprabhu, "Functionalized graphene sheets for arsenic removal and desalination of sea water," Desalination, vol. 282, pp. 39-45, 2011.

[22] Y. E. Shin, Y. J. Sa, S. Park et al., “An ice-templated, pHtunable self-assembly route to hierarchically porous graphene nanoscroll networks," Nanoscale, vol. 6, no. 16, pp. 97349741, 2014.

[23] Y. Feng, N. Feng, Y. Wei, and G. Zhang, "An in situ gelatinassisted hydrothermal synthesis of $\mathrm{ZnO}$-reduced graphene oxide composites with enhanced photocatalytic performance under ultraviolet and visible light," RSC Advances, vol. 4, no. 16, pp. 7933-7943, 2014.

[24] W. Y. Chang, Y. C. Lai, T. B. Wu, S. F. Wang, F. Chen, and M. J. Tsai, "Unipolar resistive switching characteristics of $\mathrm{ZnO}$ thin films for nonvolatile memory applications," Applied Physics Letters, vol. 92, no. 2, pp. 1-4, 2008.

[25] E. W. Lim and R. Ismail, "Conduction mechanism of valence change resistive switching memory: a survey," Electronics, vol. 4, no. 3, pp. 586-613, 2015.

[26] B. T. Phan, C. Jung, T. Choi, and J. Lee, "Trap-controlled space-charge-limited current conduction in the Cr-doped SrTiO3 thin films deposited by using pulsed laser deposition," Journal of the Korean Physical Society, vol. 51, no. 92, pp. 664668, 2007.

[27] F. C. Chiu, "A review on conduction mechanisms in dielectric films," Advances in Materials Science and Engineering, vol. 2014, 18 pages, 2014.

[28] M. A. Lampert, "Simplified theory of space-charge-limited currents in an insulator with traps," Physics Review, vol. 103, no. 6, pp. 1648-1656, 1956.

[29] S. C. Ray, "Application and uses of graphene oxide and reduced graphene oxide," in Applications of Graphene and Graphene-Oxide Based Nanomaterials, pp. 39-55, Elsevier, 2015. 